University of Nebraska - Lincoln

DigitalCommons@University of Nebraska - Lincoln

\title{
$4-2011$
}

\section{When measured spin polarization is not spin polarization}

Peter A. Dowben

University of Nebraska-Lincoln, pdowben@unl.edu

Ning Wu

University of Nebraska-Lincoln, nwu3@unl.edu

Christian Binek

University of Nebraska-Lincoln, cbinek@unl.edu

Follow this and additional works at: https://digitalcommons.unl.edu/physicsbinek

Part of the Physics Commons

Dowben, Peter A.; Wu, Ning; and Binek, Christian, "When measured spin polarization is not spin polarization" (2011). Christian Binek Publications. 72.

https://digitalcommons.unl.edu/physicsbinek/72

This Article is brought to you for free and open access by the Research Papers in Physics and Astronomy at DigitalCommons@University of Nebraska - Lincoln. It has been accepted for inclusion in Christian Binek Publications by an authorized administrator of DigitalCommons@University of Nebraska - Lincoln. 


\title{
When measured spin polarization is not spin polarization
}

\author{
P A Dowben ${ }^{1}$, Ning Wu and Christian Binek \\ Department of Physics and Astronomy and the Nebraska Center for Materials and \\ Nanoscience, University of Nebraska-Lincoln, Lincoln, NE 68588-0299, USA \\ E-mail: pdowben@unl.edu
}

Received 9 March 2011, in final form 14 March 2011

Published 8 April 2011

Online at stacks.iop.org/JPhysCM/23/171001

\begin{abstract}
Spin polarization is an unusually ambiguous scientific idiom and, as such, is rarely well defined. A given experimental methodology may allow one to quantify a spin polarization but only in its particular context. As one might expect, these ambiguities sometimes give rise to inappropriate interpretations when comparing the spin polarizations determined through different methods. The spin polarization of $\mathrm{CrO}_{2}$ and $\mathrm{Cr}_{2} \mathrm{O}_{3}$ illustrate some of the complications which hinders comparisons of spin polarization values.
\end{abstract}

What does a high polarization actually tell us when measured in spin polarized photoemission, spin polarized inverse photoemission, spin polarized metastable atom quenching or other essentially surface sensitive spectroscopies? Are the sometimes measured high polarizations truly representative of a high polarization material in a different experimental situation? Underlying these issues is the need to consider the definition of polarization; not all polarization measurements are equal [1-3]. Simplistically, the electronic bands in a ferromagnetic metal are exchange-split, and consequently the density of states at the Fermi level is different for majority and minority spin electrons, $N_{\uparrow}$ and $N_{\downarrow}$ respectively. Yet polarization depends on the measurement. We do not always measure a polarization that follows a definition of spin polarization that depends only on the population of majority and minority spin electrons, $N_{\uparrow}$ and $N_{\downarrow}$, alone. In many cases, it is not exactly clear what polarization has been measured and, as noted below, sometimes it is not even clear what material was in fact measured. Certainly, the spin polarizations derived from different experimental methodologies cannot be compared directly. For some measurements there are necessary corrections for the Fermi velocity $v_{\uparrow, \downarrow}$ to the spin polarization

\footnotetext{
1 Address for correspondence: Department of Physics and Astronomy, University of Nebraska, 855 Jorgensen Hall, North 16th Street, PO Box 880299, Lincoln, NE 68588-0111, USA.
}

$P$ which then has to be defined as:

$$
P=\frac{N_{\uparrow}\left(E_{\mathrm{F}}\right) \nu_{\uparrow}^{n} \tau_{\uparrow}^{m}-N_{\downarrow}\left(E_{\mathrm{F}}\right) \nu_{\downarrow}^{n} \tau_{\downarrow}^{m}}{N_{\uparrow}\left(E_{\mathrm{F}}\right) \nu_{\uparrow}^{n} \tau_{\uparrow}^{m}+N_{\downarrow}\left(E_{\mathrm{F}}\right) \nu_{\downarrow}^{n} \tau_{\downarrow}^{m}}
$$

where $n=1$ (and $m=0$ ) applies to the ballistic regime and $n=2$ (and $m=1$ ) applies to the diffusive regime [1-3]. Thus a large difference between the spin majority and minority Fermi velocities can in some measurements overcome an otherwise lackluster asymmetry in the spin dependent density of states.

Neglecting interfaces and other complications, the diffusive regime $P(n=2)$ should be roughly the spin polarization from a bulk conductivity measurement. This is the regime of classical Bloch-Boltzmann transport theory [4]. Here $\tau_{\uparrow, \downarrow}$ is the spin dependent relaxation time, which depends on the nature and type of the scatterers (typically defects or impurities), as well as the density of electronic states available for scattering in the crystal. The relaxation time is typically spin dependent, and should not be ignored in the diffusive spin dependent transport, so although this picture remains too simplified, the definition of spin polarization from transport measurements has $m=1(m \neq 0)$.

In the ballistic regime where $n=1$ and the spin dependent relaxation time is neglected (i.e. $m=0$ ), the polarization $P$ in equation (1) is more characteristic of tunneling current with a very thin small barrier and, in principle, Andreev reflection, as measured at a point contact between a superconductor 


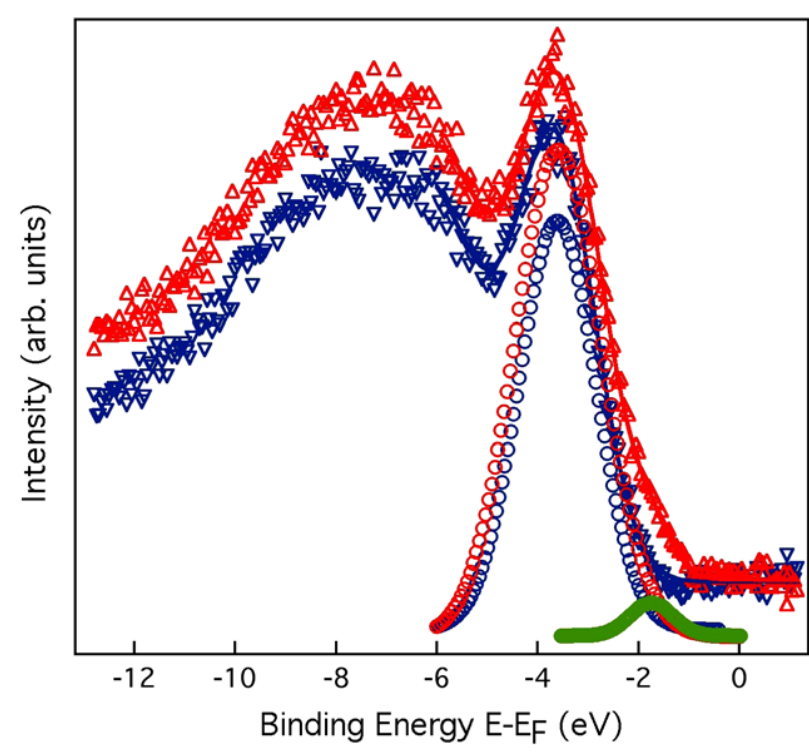

Figure 1. Spin polarized photoemission measurements from a $\mathrm{Cr}_{2} \mathrm{O}_{3}(0001)$ surface measured at $T=100 \mathrm{~K}$ after magnetoelectric cooling in $E=3.85 \times 10^{-4} \mathrm{kV} \mathrm{mm}^{-1}$ and $\mu_{0} H=30 \mathrm{mT}$ from $T>T_{\mathrm{N}}$. Spin-up and spin-down intensities are shown by red triangles and blues triangles, respectively. The open circles show $\mathrm{Cr}$ $3 \mathrm{~d}$ spin-up (red and green) and spin-down (blue) contributions at the top of the valence band where the Gaussian fit shown by the green reflects specifically the surface contribution (i.e. the surface states). Note that the density of states with strong surface weight is highly spin polarized. The photon energy is $54 \mathrm{eV}$. Adapted from [32].

and a ferromagnet $[1,2]$. In the ballistic transport regime, the current densities are uniquely defined by the electronic structure. Neither the ballistic regime $(n=1)$ nor the diffuse transport regime $(n=2)$ are really a completely accurate description of the spin polarization of the tunneling current, measured in tunneling from a ferromagnet through an insulator into a superconductor [2]. Such Tedrow and Meservey [5-7] experiments are dominated not only by polarization of the ferromagnet but also by the decay lengths of the different wavefunctions in the barrier region [8]. Even for a more simple magnetic tunnel junction, the spin polarization of the tunneling current across an insulator involves the interface states, wavevector matching, state symmetry, and differences in the wavefunction attenuation into the barrier layer. These factors, when combined, might best be described as a spin dependent interface transmission function $\left(T_{\uparrow, \downarrow}\right)$ [9]. This leads to a relationship [9]:

$$
P=\frac{T_{\uparrow}-T_{\downarrow}}{T_{\uparrow}+T_{\downarrow}} .
$$

In spin polarized photoemission, spin polarized inverse photoemission and spin polarized metastable helium atom spectroscopies, the polarization of equation (1) is measured more directly as a function of binding energy and does not require a correction for the Fermi velocity (i.e. $n=0$ ), and again the spin dependent relaxation time is typically neglected (i.e. $m=0$ ). But for these spin polarized electron spectroscopies, while the measurements may not require any correction of the Fermi velocity, surfaces do matter

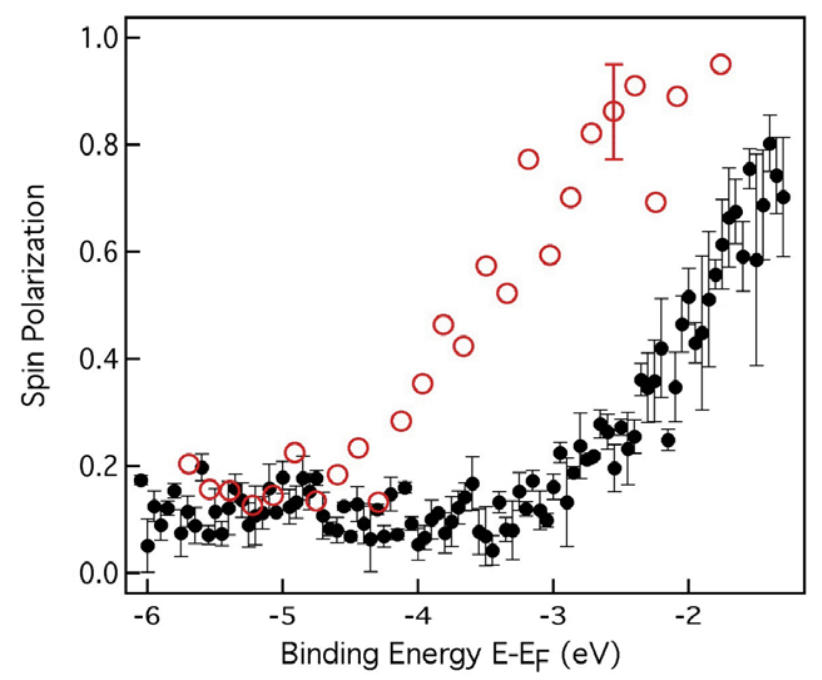

Figure 2. The polarization is plotted as extracted from the spin contrast $P=\left(I_{\uparrow}-I_{\downarrow}\right) /\left(I_{\uparrow}+I_{\downarrow}\right)$, in the spin dependent photoemission intensities versus binding energy for the $\mathrm{Cr}_{2} \mathrm{O}_{3}(0001)$ surface (black filled circles) as adapted from [32] and for $\mathrm{CrO}_{2}$ surface (red open circles) as adapted from [24], measured at $100 \mathrm{~K}$ and room temperature respectively. The experimental error in $\mathrm{P}$ are indicated by the vertical bars.

and cannot be neglected. This means that spin polarized surface states [2, 10-12] and surface composition [2, 10, 11] can affect the measurement considerably, even if the surface magnetization is stable. Further complicating interpretation, these measurements are often accompanied by very restricted wavevector sampling [11, 12]. With limited wavevector sampling, many materials will exhibit very high polarization, if only in a limited region of the Brillouin zone. Overall, it is very easy to be led astray by a measurement that suggests a very high spin polarization material.

As an example, consider chromium oxide. Through Andreev reflection [13-17] and superconducting tunnel junction [18] measurements, the chromium oxide $\mathrm{CrO}_{2}$ has provided some of the highest experimentally measured polarizations of any system, in the region of 92-98\%. Yet the measured polarizations fall rapidly as the temperature increases above $20-40 \mathrm{~K}$ for this material, reaching values that are low [3, 19-23], often less that $10 \%$ of the values measured at temperatures smaller than $2 \mathrm{~K}$. We expect this [10, 11]. Yet there are some very high polarization values measured using spin polarized photoemission at room temperature $[24,25]$. How could this be so?

One major complication that occurs in this system is that the stable surface of $\mathrm{CrO}_{2}$ appears to be $\mathrm{Cr}_{2} \mathrm{O}_{3}$ [26, 27], although other surface oxides may be possible as well. Chromia, $\mathrm{Cr}_{2} \mathrm{O}_{3}$, is a magnetoelectric antiferromagnet $[28,29]$ which can exhibit equilibrium boundary magnetization [30-33], which is coupled to the bulk antiferromagnetic order parameter. Boundary magnetization has now been demonstrably measured at the $\mathrm{Cr}_{2} \mathrm{O}_{3}(0001)$ surface using spin polarized photoemission spectroscopy [32], spin polarized inverse photoemission [33], X-ray magnetic circular dichroism [33] and magnetic force microscopy [33], and very high values of spin polarization have been observed at the valence band maximum, as seen in 
figure 1. As an insulator, $\mathrm{Cr}_{2} \mathrm{O}_{3}$ has no appreciable density of states at the Fermi level, and thus no polarization that would be seen in Andreev scattering or in a superconducting tunnel junction measurement.

In the temperature region just below the $\mathrm{Cr}_{2} \mathrm{O}_{3}$ Néel temperature $\left(T_{\mathrm{N}}=308 \mathrm{~K}\right)$, the surface spin polarization can be enhanced (and reversed) by forming a single domain antiferromagnetic state in combined applied electric and magnetic fields [32], something easily achieved in a spin polarized photoemission experiment, particularly for the surface layer. For a $\mathrm{Cr}_{2} \mathrm{O}_{3}$ surface layer on $\mathrm{CrO}_{2}$, there is the induced magnetization from the substrate and an electric field from the surface charge created at the surface of a dielectric as a result of photoemission. The combination of magnetic and electric fields may create the single domain state at the surface of $\mathrm{Cr}_{2} \mathrm{O}_{3}$ [33], with sufficient fidelity to measure a high surface spin polarization. So those high spin polarization values measured for $\mathrm{CrO}_{2}$ [24, 25], may well in fact be an elegant early experimental demonstration of boundary magnetization of an insulator that happens to be a magnetoelectric antiferromagnet, i.e. $\mathrm{Cr}_{2} \mathrm{O}_{3}$. While this is just speculation, there is something of a correspondence between the spin polarization measurements for the surface of $\mathrm{CrO}_{2}$ [24] and that of the insulator $\mathrm{Cr}_{2} \mathrm{O}_{3}$ [32], as seen in figure 2. Neither spin polarized photoemission measurement has a reliable spin polarization value near the Fermi level and both measure extremely high values of polarization at temperatures well above $4 \mathrm{~K}$.

Surfaces are often different materials, and typically have a different electronic structure from the bulk. One should be cautious about applying any surface sensitive measurement to the bulk material. This is especially true for measurements with often very limited wavevector sampling, such as photoemission and inverse photoemission, because there are materials (even non-magnetic materials like Bi and $\mathrm{Au}$ ) with very high measured polarization values but in a very limited region of the Brillouin zone. There are also some thin films where the magnetism is metastable and yet they exhibit spin polarization on the time scale of the spin polarized measurement. The polarization measurements may be performed at remanence, but are often initiated by application of a strong pulsed field. For a metastable magnetic system, there may be significant polarization if the experimental time scale $t$ is small, relative to the overall characteristic magnetization decay $\tau_{\text {mag }}$ at remanence, so that:

$$
P=P_{o} \exp \left(-t / \tau_{\mathrm{mag}}\right) .
$$

Because of the many complications in measuring polarization, as yet, there still remains no convincing experimental demonstration of extremely high polarization values in the vicinity of room temperature in any metallic system. This should not be a surprise and should be regarded as the expected outcome $[2,10,11]$.

\section{Acknowledgments}

This work was supported by NSF through Career DMR0547887, the Nebraska MRSEC (DMR-0820521), and by the NSF/SRC-NRI supplement to the Nebraska MRSEC. The authors would like to thank $\mathrm{Xi} H \mathrm{He}$ for his technical assistance in making this work possible, as well as acknowledge helpful discussions with Evgeny Tsymbal and Kirill Belashchenko.

\section{References}

[1] Mazin I I 1999 Phys. Rev. Lett. 831427

[2] Velev J P, Dowben P A, Tsymbal E Y, Jenkins S J and Caruso A N 2008 Surf. Sci. Rep. 63 400-25

[3] Coey J M D and Venkatesan M 2002 J. Appl. Phys. 918345

[4] Allen P B 1978 Phys. Rev. B 173725

[5] Tedrow P M and Meservey R 1971 Phys. Rev. Lett. 26192

[6] Tedrow P M and Meservey R 1973 Phys. Rev. B 7318

[7] Meservey R and Tedrow P M 1994 Phys. Rep. 238173

[8] Slonczewski J C 1989 Phys. Rev. B 396995

[9] Belashchenko K D, Tsymbal E Y, van Schilfgaarde M, Stewart D A, Oleynik I I and Jaswal S S 2004 Phys. Rev. B 69174408

[10] Dowben P A and Skomski R 2004 J. Appl. Phys. 95 7453-8

[11] Dowben P A and Jenkins S J 2005 The limits to spin-polarization in finite-temperature half-metallic ferromagnets Frontiers in Magnetic Materials ed A Narlikar (Berlin: Springer) pp 295-325

[12] Skomski R and Dowben P A 2002 Europhys. Lett. 58 544-8

[13] Anguelouch A, Gupta A, Xiao G, Miao G X, Abraham D W, Ingvarsson S, Ji Y and Chien C L 2002 J. Appl. Phys. 91 7140-2

[14] Ji Y, Strijkers G J, Yang F Y, Chien C L, Byers J M, Anguelouch A, Xiao G and Gupta A 2001 Phys. Rev. Lett. 86 5585-8

[15] Soulen R J Jr et al 1998 Science 28285

[16] Soulen R J et al 1999 J. Appl. Phys. 854589

[17] DeSisto W J, Broussard P R, Ambrose T F, Nadgomy B E and Osofsky M S 2000 Appl. Phys. Lett. 763789

[18] Parker J S, Watts S M, Ivanov P G and Xiong P 2002 Phys. Rev. Lett. $\mathbf{8 8} 196601$

[19] Barry A, Coey J M D and Viret M 2000 J. Phys.: Condens. Matter 12 L173

[20] Manoharan S S, Elefant D, Reiss G and Goodenough J B 1998 Appl. Phys. Lett. 72984

[21] Watts S M, Wirth S, von Molnár S, Barry A and Coey J M D 2000 Phys. Rev. B 619621

[22] Coey J M D, Berkowitz A E, Balcells L, Putris F and Barry A 1998 Phys. Rev. Lett. 803815

[23] Hwang H Y and Cheong S W 1997 Science 2781607

[24] Kämper K P, Schmitt W, Güntherodt G, Gabino R J and Ruf R 1987 Phys. Rev. Lett. 592788

[25] Dedkov Yu S, Fonine M, König C, Rüdiger U, Güntherodt G, Senz S and Hesse D 2002 Appl. Phys. Lett. 804181

[26] Cheng R, Xu B, Borca C N, Sokolov A, Yang C-S, Yuan L, Liou S-H, Doudin B and Dowben P A 2001 Appl. Phys. Lett. 79 3122-4

[27] Dai J, Tang J, Xu H, Spinu L, Wang W, Wang K-Y, Kumbhar A, Li M and Diebold U 2000 Appl. Phys. Lett. 772840

[28] Borisov P, Hochstrat A, Shvartsman V V and Kleemann W 2007 Rev. Sci. Instrum. 78106105

[29] Fiebig M 2005 J. Phys. D: Appl. Phys. 38 R123-52

[30] Andreev A F 1996 JETP Lett. 63758

[31] Belashchenko K D 2010 Phys. Rev. Lett. 105147204

[32] He Xi, Wang Yi, Wu N, Caruso A N, Vescovo E, Belashchenko K D, Dowben P A and Binek C 2010 Nat. Mater. 9 579-85

[33] Wu N, He Xi, Wysocki A, Lanke U, Komesu T, Belashchenko K D, Binek C and Dowben P A 2011 Phys. Rev. Lett. 106087202 